COMMENT

\title{
Factors influencing mortality estimates in post-release survival studies
}

\author{
Michael K. Musyl ${ }^{1, *}$, Christopher D. Moyes ${ }^{2}$, Richard W. Brill ${ }^{3}$, Nuno M. Fragoso ${ }^{4}$ \\ ${ }^{1}$ University of Hawaii, Joint Institute for Marine and Atmospheric Research (JIMAR), Kewalo Research Facility/NOAA, \\ 1125B Ala Moana Boulevard, Honolulu, Hawaii 96814, USA \\ ${ }^{2}$ Department of Biology, Queen's University, Kingston, Ontario, K7L 3N6, Canada \\ ${ }^{3}$ Cooperative Marine Education and Research Program, NMFS-NEFSC, Virginia Institute of Marine Science, PO Box 1346, \\ Gloucester Point, Virginia 23062, USA \\ ${ }^{4}$ Large Pelagics Research Center, University of New Hampshire, Durham, New Hampshire 03824, USA
}

\begin{abstract}
Campana et al. (2009; Mar Ecol Prog Ser 387:241-253) explored the survival of blue sharks Prionace glauca captured and released from the North Atlantic commercial longline fishery. We think that their comments and comparisons do not accurately reflect a previous survival study of blue sharks in Hawaii (Moyes et al. 2006; Trans Am Fish Soc 135:1389-1397). The differences in mortality between the studies, $\sim 5 \%$ in the Hawaii-based fishery and $\sim 35 \%$ in the North Atlantic fishery, were suggested to be due to failure of Moyes et al. (2006) to accurately reflect commercial fishing conditions. Careful examination of the data, however, suggests that the mortality depends on fisheryspecific features - hook type, soak time and handling of the bycatch during release — rather than the respective tagging protocols. Survival studies based on pop-up satellite archival tags (PSATs) are cost-prohibitive; alternative (e.g. biochemical) approaches to estimate stress and morbidity are needed to supplant PSAT studies and to increase sample sizes. Standardization of fishing methods and sampling protocol is needed for future survival studies, to reduce experimental bias and improve the cost:benefit relationship.
\end{abstract}

KEY WORDS: Pop-up satellite archival tag $\cdot$ PSAT $\cdot$ Hook type $\cdot$ Soak time $\cdot$ Bycatch handling trauma Longline fishery

Resale or republication not permitted without written consent of the publisher

\section{Introduction}

Management strategies for mitigating effects on the bycatch of large-scale commercial fisheries require accurate estimates of post-release survival in captured teleosts, elasmobranchs and sea turtles. The recent study by Campana et al. (2009) offers some interesting observations on mortality in the North Atlantic commercial longline fishery, which targets swordfish Xiphias gladius and in which blue sharks Prionace glauca are the principal bycatch. The conclusions by Campana et al. differ in many respects from the survival study by Moyes et al. (2006) on blue sharks released from pelagic longline fishing gear targeting swordfish in Hawaii. Both studies employed pop-up satellite archival tags (PSATs) to assess postrelease mortality. The main difference between the 2 studies is the much greater mortality in Campana et al. $(\sim 35 \%)$ than in Moyes et al. $(\sim 5 \%)$, in estimates both from observer studies and PSATs. This Comment proposes some unifying themes, and clarifies important factors used to interpret results from survival studies, in greater detail than was possible in Campana et al. 


\section{Bias in tagging}

As discussed in both Moyes et al. (2006) and Campana et al. (2009), the choice of which individuals to tag inevitably introduces bias that must be considered when extrapolating experimental results to the commercial fishery. Moyes et al. did not tag sharks that were dead or apparently moribund (i.e. lethargic and unresponsive to handling). Still, one PSAT-tagged blue shark ultimately died post-release. In contrast, Campana et al. (p. 243) tagged 'a random sample of 40 sharks (both injured and healthy)'. While our choice would appear to introduce some bias, we reported that only $5 \%$ of the captured sharks were categorized as dead or moribund in Moyes et al. Thus, shark mortality (acute or delayed) in the Hawaii-based fishery for swordfish was underestimated at most by $5 \%$. The estimate in Moyes et al., based on 9 moribund individuals of a total of 172 blue sharks captured, has recently been confirmed in a larger scale study by Walsh et al. (2009), who estimated a minimum mortality of 4.0 to $8.5 \%$ for blue sharks, in contrast to $>20 \%$ for other shark species.

Could differential mortality between the 2 studies have been influenced by shark size? Campana et al. (2009; p. 245) used different hook types and sizes and reported that smaller sharks were 'more likely to be retrieved dead from the hook', but they did not report the sizes of PSAT-tagged sharks that ultimately died. Moyes et al. (2006) used similar gear and fished at the same time and locations as the commercial longline fleet. As a result, blue sharks tagged and sampled in Moyes et al. had a mean fork length (FL) of $\sim 170 \mathrm{~cm}$ (M. Musyl, unpubl. data), which was similar to the mean size in the fishery (Walsh et al. 2009). No significant differences could be demonstrated between biochemical correlates of stress and morbidity and shark size (C. Moyes et al., unpubl. data).

\section{Capture and tagging stress}

In any study assessing the effect of the fishing technique on post-release survival, the goal is to mimic the practices of the commercial fishery. Campana et al. $(2009$; p. 250) suggested that Moyes et al. may have found reduced mortality because we captured the sharks on a 'scientific research cruise', but we specifically designed our tagging approach to mimic the fishing and release practices used in the commercial fishery.

(1) Fishing techniques. In the North Atlantic fishery, Campana et al. (2009) and Carruthers et al. (2009) observed that fishing with $\mathrm{J}$ hooks caused much higher mortality, as a result of ingestion of the hook. The
Hawaii-based fishery and Moyes et al. (2006) rely on circle hooks, or Japanese ringed hooks, which cause much less injury ( $\mathrm{J}$ hooks have been banned in the fishery since 2004; R. Ito, pers. comm.). In addition to fishing gear, Moyes et al. also used soak times (time during which the gear is in the water; 10 to $18 \mathrm{~h}$ ), nighttime sets (gear deployed at dusk and retrieved at dawn), number of hooks between floats (4 to 5), and bait (squid Illex spp.) (now banned) that accurately reflect those of the Hawaii-based fishery.

(2) Release techniques. In the Campana et al. (2009) study, the sharks were probably handled more vigorously (cf. p. 251) than in Moyes et al. (2006). In addition to body gaffing, hooks were removed forcefully from the shark, occasionally leading to detachment of the jaw. In the Hawaii-based fishery, unwanted sharks are quickly released by cutting lines, leaders or hooks - sharks are not gaffed and hoisted aboard, to avoid wasting time while the crew processes the target species. It is therefore logical that the mortality of sharks should be greater in the North Atlantic fishery.

(3) Tagging. The handling of the sharks was not described in detail in Campana et al. (2009); in Moyes et al. (2006, p. 1390) we stated that the sharks were 'were brought on board in a sling and were restrained by crew with mattresses.' Though Campana et al. completed tagging within 3 min of handling, we required additional time to hoist the shark aboard the vessel to collect blood samples and affix PSATs. Thus, in contrast to the opposite suggestion by Campana et al. (p. 250: 'At a minimum, commercially caught sharks can be expected to have spent longer on the hook, and to be treated considerably less gently than those caught for scientific purposes'), handling stress in our study was probably much greater than in the Hawaii-based commercial fishery (e.g. one PSAT-tagged shark probably died as the direct result of scientific handling, as discussed in Moyes et al.).

\section{Novel approaches}

The cost of PSATs precludes their wide application in survival studies (Moyes et al. 2006, Campana et al. 2009, and references therein). Just after Campana et al. initiated their tagging work (in 2005), Moyes et al. introduced a biochemical approach to measure correlates of morbidity and mortality instead of using PSATs. A biochemical approach reduces experimental bias and increases sample size (i.e. more animals could be sampled, regardless of condition) and would therefore optimize experimental design (increase statistical power) and cost:benefit (e.g. once the method 
is operational, about 40 samples can be assayed for the cost of one PSAT [ US\$ 4000]). Though Campana et al. were not in a position to benefit from Moyes et al. during the early phases of their data collection, they could have represented the various studies with biochemical indices more accurately; instead, they stated that 'Since biochemical indices of capture stress have not been reported for blue sharks ...', and 'Biochemical indices of the stress of capture have been characterized in one shark species' (p. 251), although the references they cite include studies on several species (blue shark: Moyes et al. 2006; many pelagic teleosts and sharks: Skomal 2007; sandbar shark: Brill et al. 2008). Furthermore, Moyes et al. reported additional studies that have used biochemical approaches to assess capture stress.

\section{Final remarks}

Comparisons between different survival studies require standardization of methods, both in the fisheries and in the sampling programs. In Moyes et al. (2006) and Campana et al. (2009), the fishing techniques (hook type, soak time, and handling during release) appear to be the main factors affecting postrelease mortality of blue sharks, and their post-release fate appears to be much more favorable in the Hawaiibased fishery than in the North Atlantic fishery.

Editorial responsibility: Matthias Seaman, Oldendorf/Luhe, Germany
Acknowledgements. This project was funded by Cooperative Agreements NA37RJ0199 and NA67RJ0154 of the National Oceanic and Atmospheric Administration (NOAA) with the Joint Institute for Marine and Atmospheric Research (JIMAR), University of Hawaii. We thank B. Walsh (JIMAR), R. Ito (Pacific Islands Fisheries Science Center, NOAA, Honolulu, Hawaii) and L. McNaughton (JIMAR) for providing comments on an earlier draft.

Disclaimer. The views expressed herein are those of the authors and do not necessarily reflect the views of their agencies.

\section{LITERATURE CITED}

Brill R, Bushnell P, Schroff S, Seifert R, Galvin M (2008) Effects of anaerobic exercise accompanying catch-andrelease fishing on blood-oxygen affinity of the sandbar shark (Carcharhinus plumbeus, Nardo). J Exp Mar Biol Ecol 354:132-143

Campana SE, Joyce W, Manning MJ (2009) Bycatch and discard mortality in commercially caught blue sharks Prionace glauca assessed using archival satellite pop-up tags. Mar Ecol Prog Ser 387:241-253

Carruthers EH, Schneider DC, Neilson JD (2009) Estimating the odds of survival and identifying mitigation opportunities for common bycatch in pelagic longline fisheries. Biol Conserv 142:2620-2630

Moyes CD, Fragoso N, Musyl MK, Brill RW (2006) Predicting postrelease survival in large pelagic fish. Trans Am Fish Soc 135:1389-1397

Skomal GB (2007) Evaluating the physiological and physical consequences of capture on post-release survivorship in large pelagic fishes. Fish Manag Ecol 14:81-89

Walsh WA, Bigelow KA, Sender KL (2009) Decreases in shark catches and mortality in the Hawaii-based longline fishery as documented by fishery observers. Mar Coastal Fish 1: $270-282$

Submitted: August 20, 2009; Accepted: November 24, 2009 Proofs received from author(s): December 1, 2009 